\title{
Disruption Avoidance Through the Prevention of NTM Destabilization in TCV
}

\author{
U. A. Sheikh ${ }^{1}$, B. P. Duval ${ }^{1}$, C. Galperti ${ }^{1}$, M. Maraschek ${ }^{2}$, \\ O. Sauter ${ }^{1}$, C. Sozzi ${ }^{3}$, G. Granucci ${ }^{3}$, M. Kong ${ }^{1}$, B. Labit ${ }^{1}$, \\ A. Merle ${ }^{1}$, N. Rispoli ${ }^{3}$, the TCV Team ${ }^{1}$ and the EUROfusion \\ MST1 Team ${ }^{4}$ \\ 1 Swiss Plasma Center, École Polytechnique Fédérale de Lausanne, Lausanne, \\ Switzerland \\ 2 Max Planck Institute für Plasmaphysik, Garching, Germany \\ 3 Istituto di Fisica del Plasma - Universita degli Studi di Milano-Bicocca, Milan, Italy \\ 4 Author list of H. Meyer et al., Nuclear Fusion FEC 2016 Special Issue (2017) \\ E-mail: umar.sheikh@epfl.ch
}

March 2018

\begin{abstract}
Destabilization of a stationary neoclassical tearing mode due impurity influx can lead to a potentially destructive disruption and is of significant concern for current and future tokamaks. A representative scenario was developed on TCV to experiment with applicable disruption avoidance techniques and produce a real time control system capable of handling such an event. Soft x-ray (SXR) radiation intensity and magnetic diagnostics analyses available in real time were used to provide plasma state information to the control system. Electron cyclotron current drive (ECCD) was employed to prevent NTM destabilization. Deposition of ECCD near the calculated $\mathrm{q}=2$ surface was able to prevent destabilization of the NTM if a large increase in SXR radiation intensity was used as the trigger. A delay in avoidance resulted in the plasma entering a disruptive state which required over $100 \mathrm{~ms}$ of continuous ECCD around the $\mathrm{q}=2$ surface to stabilize. Ramp down scenarios were studied to complete the design of a closed loop system. This system was then successfully tested using increasingly disruptive scenarios, through increased gas quantities, and the system was able to extend the discharge for a prescribed amount of time and safely ramp down the plasma current to the minimum controllable level. The system demonstrated in this work is presently limited to this specific type of disruption but this approach could be applied to other disruptive situations on the path to building a global disruption handling system.
\end{abstract}

Keywords: Disruption avoidance, NTMs, TCV 


\section{Introduction}

Regimes that operate with high performance are required to produce desirable fusion yields. A limiting factor in achieving the maximum possible plasma pressure is the onset of neoclassical tearing modes (NTMs). NTMs are islands mainly driven by the loss of neoclassical bootstrap current. They cause significant degradation in confinement and can lead to complete plasma current disruptions that can damage or even destroy parts of the machine structure. To this end, significant effort is being expended by the fusion community to create a path-oriented approach to predict, identify before and react to potential disruptions $[1,2,3,4]$.

An efficient disruption handling system requires three main components; disruption identification, avoidance strategies and mitigation schemes. The identification system needs to predict the proximity to possible disruptions, provide information on the type of instability that may cause the disruption and the estimated time available to react. This information allows the control system to select the most appropriate avoidance procedure and, if it is possible to act within the available time. An avoidance oriented approach has numerous advantages over mitigation such as the possibility of continuing the discharge, diminishing any post discharge recovery resulting, say, from massive impurity injection and avoiding significant stresses on the vessel and thermal loads on plasma facing components that can occur even during a well mitigated disruption.

Automated analyses of large sets of plasma discharges and intentional creation of disruptive scenarios are commonly applied techniques to study disruptions. Automated analysis is an excellent resource to study the causes of disruptions, their likelihood and the time scales upon which these events happen over the large multi-machine datasets available. On the other hand, this approach does not allow for systematic studies of disruption avoidance or mitigation for specific types of disruptions and therefore dedicated discharges are required to develop specific techniques. The goal of this work was to expand the current knowledge on disruption avoidance and handling through the creation of a disruptive scenario upon which various avoidance techniques could be tested. An additional outcome of this work is an extended dataset allowing specific disruption identifiers to be studied in detail.

The disruptive scenario selected for this work was an impurity influx into a plasma already sustaining a saturated NTM. This scenario was selected as long duration NTMs are often observed in high performance regimes and therefore priority for the ITER project [5]. It is highly probable that NTMs will be observed in ITER as experiments attempt to achieve high performance and where changes in plasma conditions may lead to further growth of these modes. One such change in plasma conditions can result from an influx of an impurity from the machine wall or otherwise. Unchecked, such an event may cause the mode amplitude to grow, leading to a decrease in mode frequency through stronger interaction with the vessel wall and finally a locked-mode induced disruption. This study provides indicators to predict this situation and possible actions that can be carried out to avoid a disruption and optimally terminate the discharge. 
Numerous studies have been performed to control and suppress NTMs [6, 7, 8, 9, $11,12,13]$. These studies have attempted to modify the density, pressure and current profiles to replace the missing bootstrap current $[14,15]$ in the O-point of the island $[16,17]$. Electron cyclotron current drive (ECCD) using high power gyrotrons has been experimentally shown to be an efficient method to act upon these types of disruptions $[18,19,20,21,22]$. ECCD is the preferred approach due to the fast reaction times possible as well as the ability to deposit heat and generate current at locations with an accuracy of a few centimetres. ECCD was selected as the primary actuator for this study due to these reasons and a well established high power X2 control system available on TCV.

Studies of NTM stabilization through temporal power modulation to target the Opoint have been conducted on a range of machines [23, 10, 24]. Experiments on Asdex Upgrade (AUG) and JT-60 have shown improved suppression efficiency when depositing ECCD only into the O-point of the island [25, 26]. Recent experiments on DIII-D used the most advanced NTM stabilization techniques currently available; resistive magnetic perturbations to entrain a mode, preventing locking to the vessel and establishing a target for modulated ECCD deposition into the O-point [10]. These experiments were not able to achieve the desired result due to technical difficulties. Comparatively, the work presented in the paper investigates the applicability of a simpler, continuous wave ECCD deposition system for NTM regularization. Such a system would be advantageous for ITER as it would significantly reduce technical requirements. It is also shown that this is required near disruptions and with unhealthy plasmas, since the mode location and frequency are hard to determine.

\section{Experiment Description}

TCV is a medium sized tokamak with a major radius of $0.88 \mathrm{~m}$, a minor radius of $0.25 \mathrm{~m}$ and a maximum toroidal field of $1.5 \mathrm{~T}$. The combination of electron cyclotron resonance heating $(\mathrm{ECRH})$ systems, a versatile RT control system, wide array of diagnostics, a fast gas injection valve and carbon walls that are relatively unaffected by disruptions make TCV an ideal machine to study disruption avoidance techniques. The second harmonic (X2) ECRH system consists of four independently steerable mirrors with a total potential peak power of $2.5 \mathrm{MW}$, which can be delivered as only heating or accompanied by current drive through variations in toroidal angle. The power delivered and deposition location of each launcher can be commanded in RT by the digital control system or follow a preprogrammed trajectory.

The distributed digital RT control system on TCV is a highly modular structure communicating over reflective-memory that allows for the addition of machine nodes. This enables controllers to be developed independently and be integrated into the multicontroller environment. Three such systems were enhanced and integrated for this study: RT magnetics analysis to identify the presence of MHD modes, RT-TORBEAM for ECCD launcher control [27, 28] and quasi in-line electron cyclotron emission (QI- 
ECE) to track and target an NTM of sufficient amplitude [29]. These systems employed the RT-LIUQE code [30] that provides RT equilibrium reconstructions with a temporal resolution better than one $\mathrm{ms}$ [31].

Figure 1 illustrates the layout of TCV's coils and magnetic probes. The magnetic diagnostic system consists of flux loops mounted outside the vessel and magnetic field probes and saddle loops mounted inside the vessel. Four poloidal arrays of 38 magnetic field probes are used to measure the time derivative of the magnetic field tangential to the vessel $\left(\dot{B}_{p o l}\right)$. There are 24 saddle loops measuring the time derivative of the radial magnetic flux enclosed by the loop surface $\left(\dot{B}_{r}\right)$ and are placed on a $3 \times 8$ grid covering toroidal angles of $45^{\circ}$ each at three heights on the vacuum vessel. Finally, poloidal fluxes are measured by 61 flux loops that are placed close to the poloidal field coils. The temporal resolution of the system varies from $250 \mathrm{kHz}$ for the magnetic field probes to $5 \mathrm{kHz}$ for the saddle loops. The plasma current is obtained by trapezoidal integration of the discrete magnetic probe measurements and the total magnetic energy of the plasma is measured by a diamagnetic loop [32].

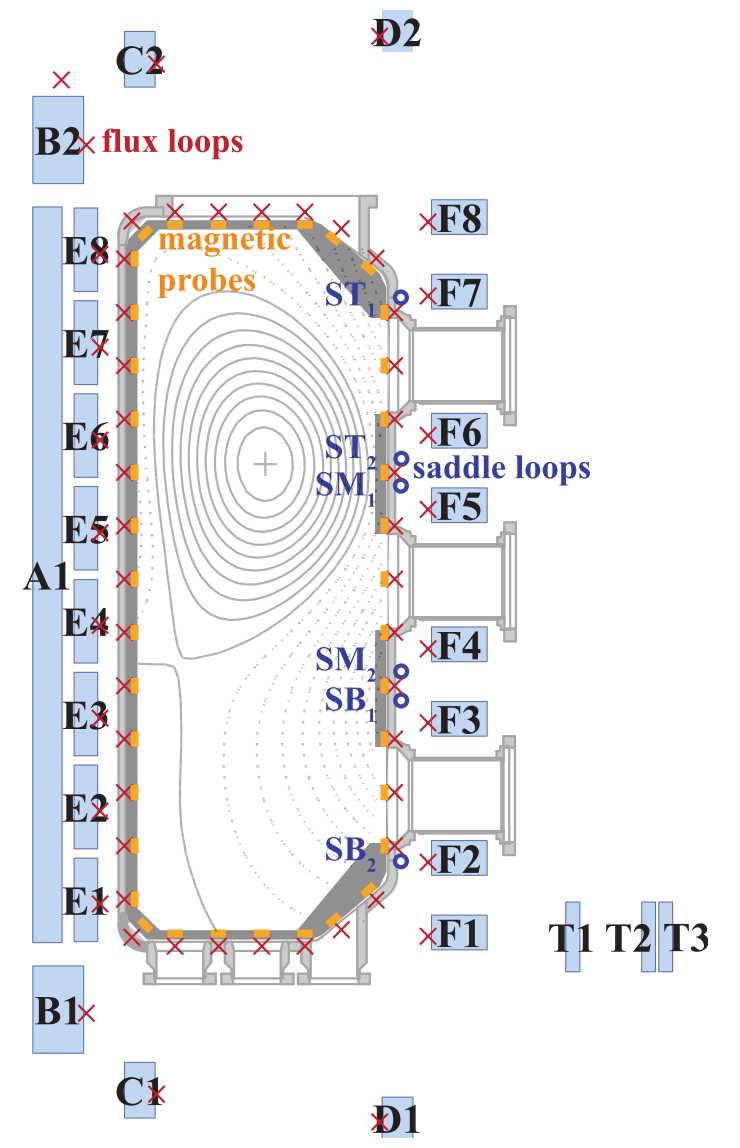

Figure 1. TCV poloidal cross section with a typical plasma equilibrium showing the ohmic transformer coils A, B, C and D, the shaping coils E and F, the toroidal field coil connections $\mathrm{T}$, the poloidal flux loops (red crosses), the magnetic field probes measuring $\dot{B}_{p o l}$ (orange rectangles) and the saddle flux loops measuring $\dot{B}_{r}$ (blue circles). [33].

The primary radiation diagnostics used in this study were the filtered photodiodes, 
XTOMO soft X-ray (SXR) diodes and the Duplex Multiwire Proportional SXR counter (DMPX). The filtered photodiodes view the plasma across a vertical line of sight at the radial centre of the tokamak with a temporal resolution of $50 \mathrm{kHz}$. The two diodes utilized in the study were filtered to measure D-alpha emission at $656.3 \mathrm{~nm}$ and He-II emission at $468.6 \mathrm{~nm}$. The XTOMO diagnostic consists of 200 lines of sight with vertical and horizontal coverage of the entire vessel allowing for tomographic reconstructions. The XTOMO diodes are sensitive to photons of energies between $1 \mathrm{keV}$ and $10 \mathrm{keV}$ and have a temporal resolution of $100 \mathrm{kHz}$. The DMPX diagnostic has 32 vertical lines of sight, as shown in Figure 2, and can also be inverted with the assumption of cylindrical symmetry to provide SXR radial emission profiles. This system is sensitive to photons of energies between 3 and $30 \mathrm{keV}$ and features a temporal resolution of $200 \mathrm{kHz}$.

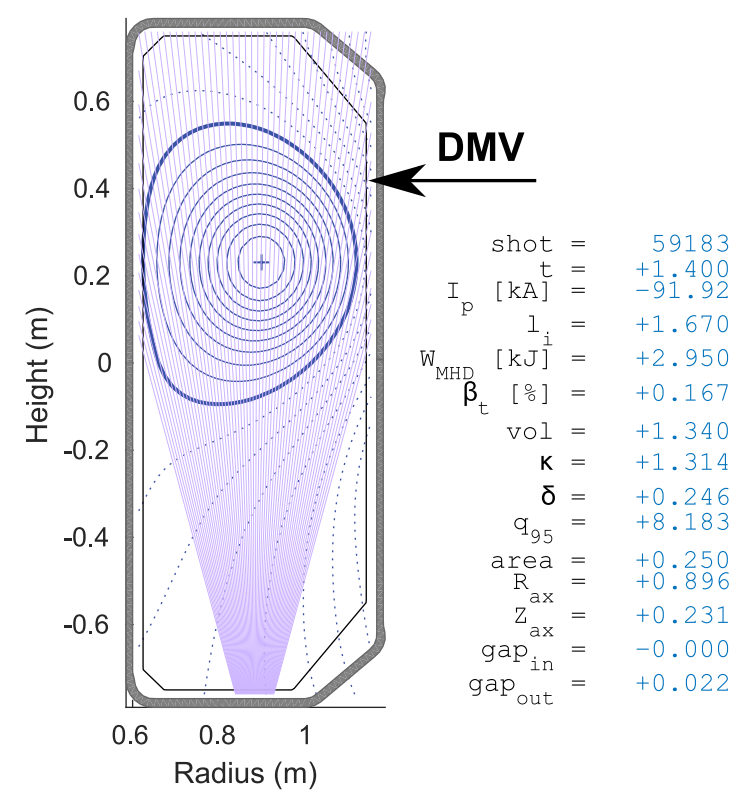

Figure 2. Plasma shape overlaid with lines of sight of the DMPX diagnostic, reference discharge parameters and relative location of the DMV used to inject neon.

\subsection{Experiment Scenario}

A limited L-mode plasma with the shape and global parameters presented in Figure 2 was used for these experiments. X2 gyrotrons with a nominal power of 1.0 MW delivered a calculated $35 \mathrm{kA}$ of on-axis co-current ECCD to induce a saturated NTM [13]. The plasma remained in this stationary state until neon was injected through a fast valve at $1.5 \mathrm{~s}$ to simulate an impurity influx. The amount of injected gas was experimentally varied to find a flux that would cool the plasma and further destabilize the NTM but not cause an immediate disruption, thus allowing sufficient time to experiment with disruption avoidance techniques. The required quantity was approximately $10 \%$ of the total plasma bulk $\left(2 \times 10^{18}\right.$ particles $/ 0.08$ mbar L) injected in $25 \mathrm{~ms}$ through the 
disruption mitigation valve (DMV) mounted on an upper lateral port.

A windowed Fourier spectrogram of the MHD activity measured on a $\dot{B}_{\text {pol }}$ probe for a reference discharge in which no avoidance action was taken is presented in Figure 3. MHD analysis identified the initial saturated NTM to be a 2/1 mode with a rotation frequency of $3 \mathrm{kHz}$. The injection of neon at $1.5 \mathrm{~s}$ resulted in the core electron temperature dropping from approximately $2.0 \mathrm{keV}$ to $750 \mathrm{eV}$ and broadening of the qprofile. The plasma re-stabilized approximately $20 \mathrm{~ms}$ after the gas injection and a saturated 2/1 NTM with a relatively increased amplitude and a frequency of $7.5 \mathrm{kHz}$ was observed. The discharge remained stable for a further $40 \mathrm{~ms}$ and at $1.56 \mathrm{~s}$, the mode amplitude grew quickly and the frequency decreased leading to a disruptive, unstable NTM. This was followed by an extended disruptive phase lasting $0.7 \mathrm{~s}$, during which the NTM repeatedly reformed as the plasma recovered, grew quickly in amplitude and caused another minor disruption.

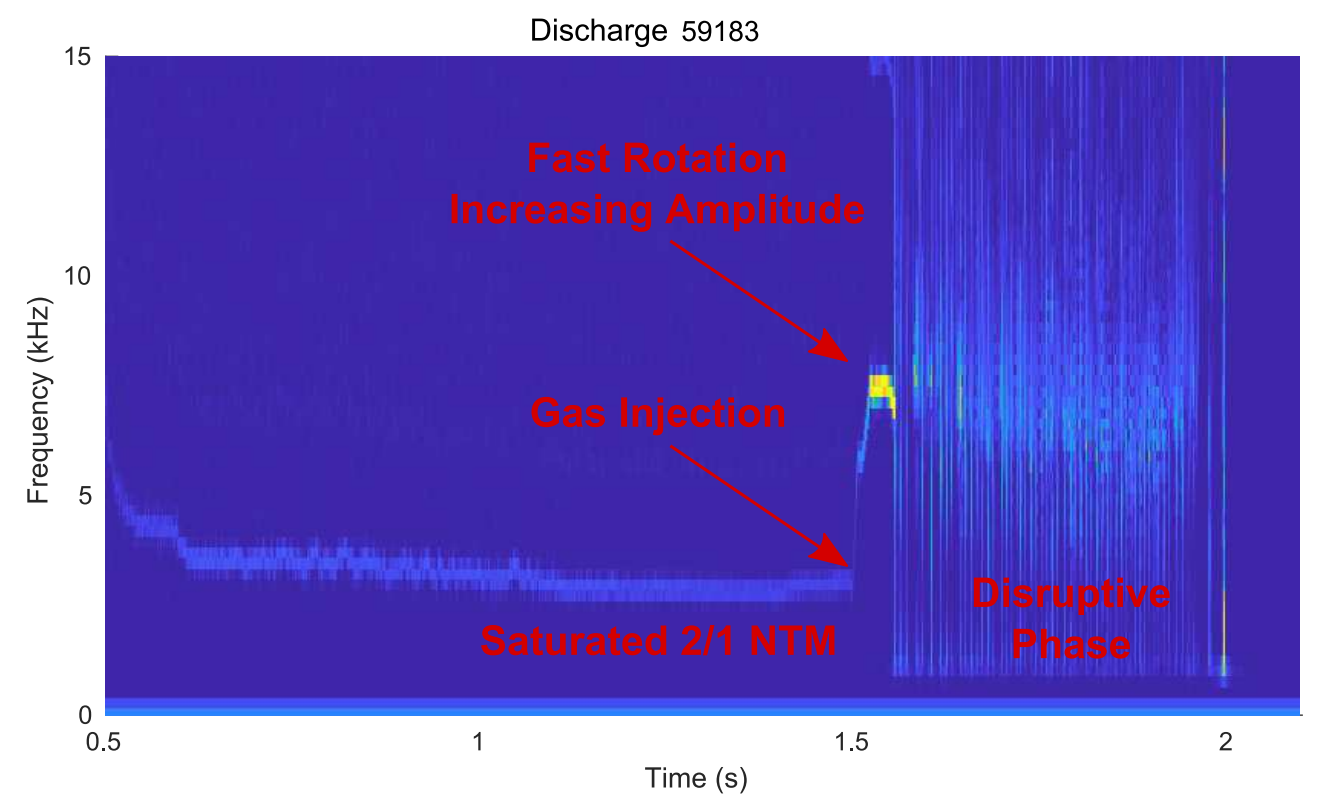

Figure 3. Windowed Fourier spectra of MHD activity measured at a high field side magnetic flux probe closest to the magnetic axis.

The NTM increased in amplitude and caused the minor disruption in approximately $0.5 \mathrm{~ms}$ from the $\dot{B}_{\text {pol }}$ measurements presented in Figure 4. The sudden mode amplitude growth caused a $70 \%$ reduction in $T_{e}$ within $0.4 \mathrm{~ms}$ and an increase in the D-alpha and He-II photodiode signals. The resulting first minor disruption at $1.5595 \mathrm{~s}$ resulted in a fast decrease in the normalised inductance and core current density. The drop in normalised inductance and core current density broadened the current profile that is illustrated in the LIUQE reconstructed current density profiles shown in Figure 5. The profiles indicate an increasing peaking of the current profile for approximately $50 \mathrm{~ms}$ after neon is injected. The fast current profile flattening also results in an increased plasma volume, also observed in Figure 4. This initial minor disruption pushes the 
plasma into a disruptive phase during which the profile repeatedly recovers and crashes.
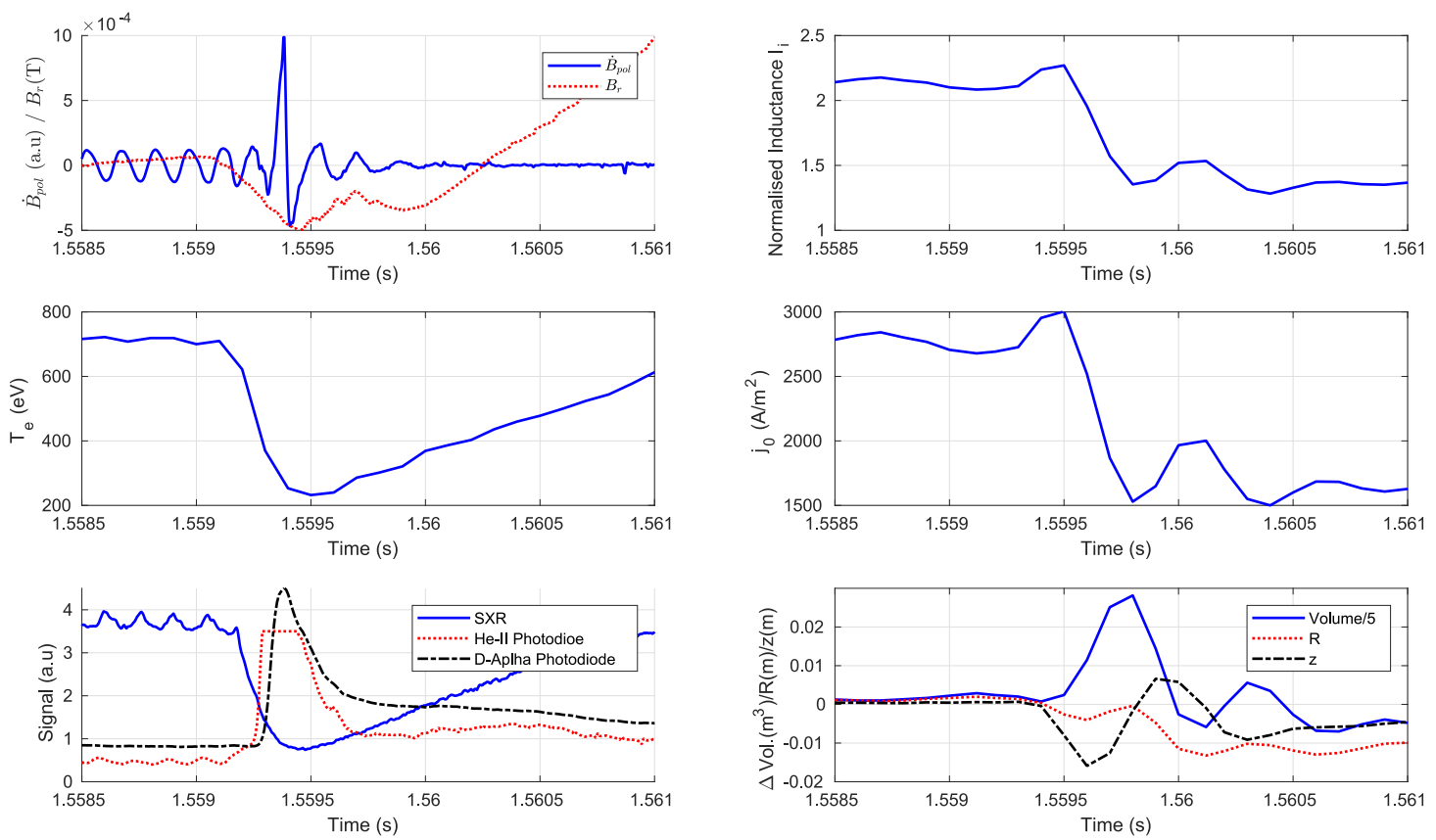

Figure 4. Measurements from magnetic probes, core $T_{e}$ from the xTe diagnostic, SXR emission and filtered photodiodes for discharge 59183. Reconstructed normalised inductance $\left(l_{i}\right)$, core current density $\left(j_{0}\right)$, plasma volume and magnetic axis position from LIUQE.

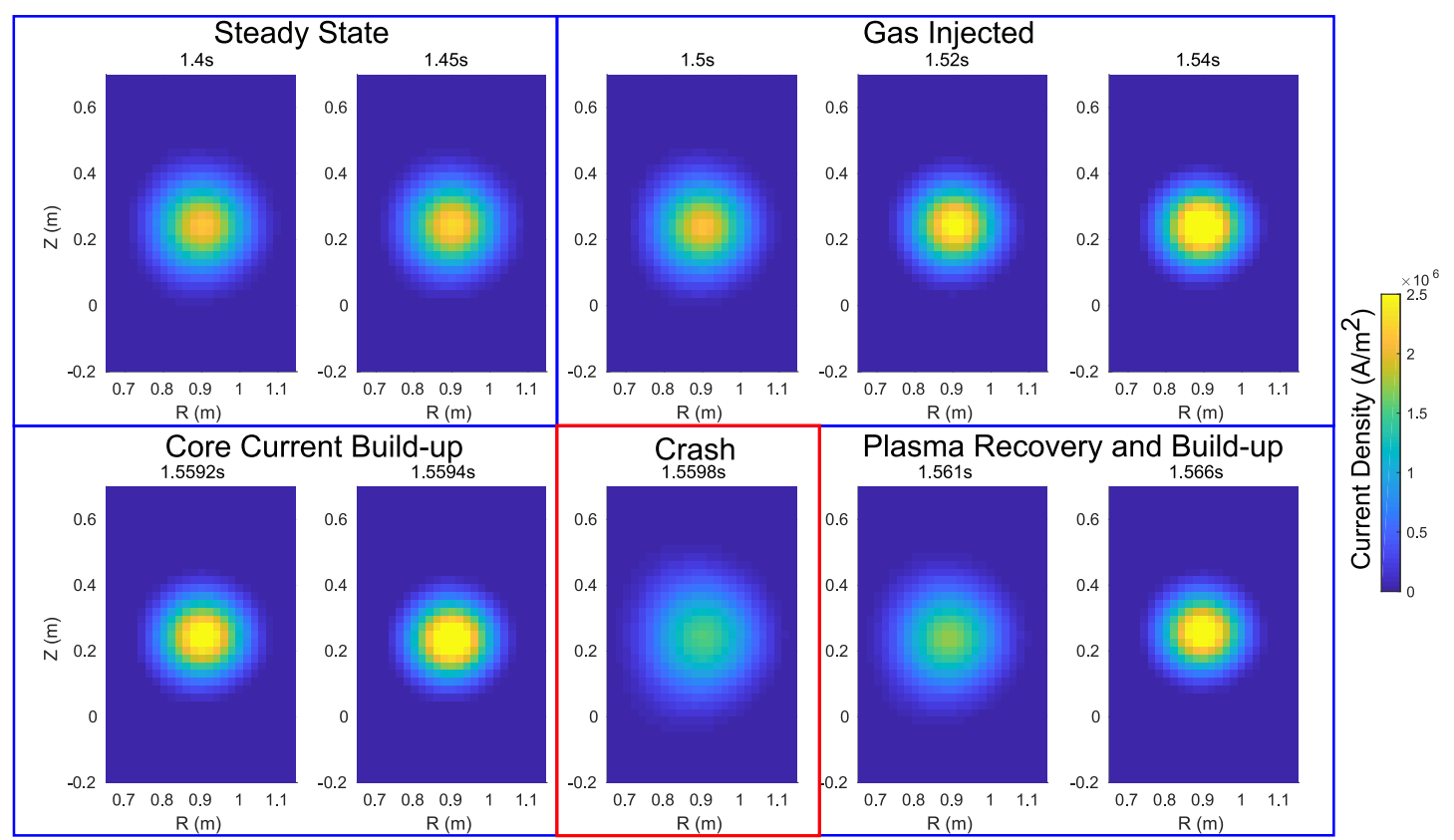

Figure 5. Current density profiles reconstructed by LIUQE for discharge 59183. 
The SXR inversion and filtered photodiode signals presented in Figure 6 indicate two types of minor disruptions occurring during this disruptive phase of the discharge. Prior to the initial minor disruption, the SXR inversion confirms the presence and frequency of the 2/1 NTM inferred from the magnetics analysis. A significant reduction in SXR intensity is observed across the entire profile $0.4 \mathrm{~ms}$ before the minor disruption. This is followed by a spike in the D-alpha and He-II photodiode signals indicating the minor disruption reaches the plasma boundary. The second minor disruption observed exhibits a different radiative signature; the decrease in SXR radiation remains primarily in the core and no spike in the D-alpha emission occurs. This indicates that the second minor disruption was considerably more internal to the plasma and did not reach the edge of plasma where it would have affected the D-alpha intensity. The two events will be refereed to as large and small minor disruptions respectively.

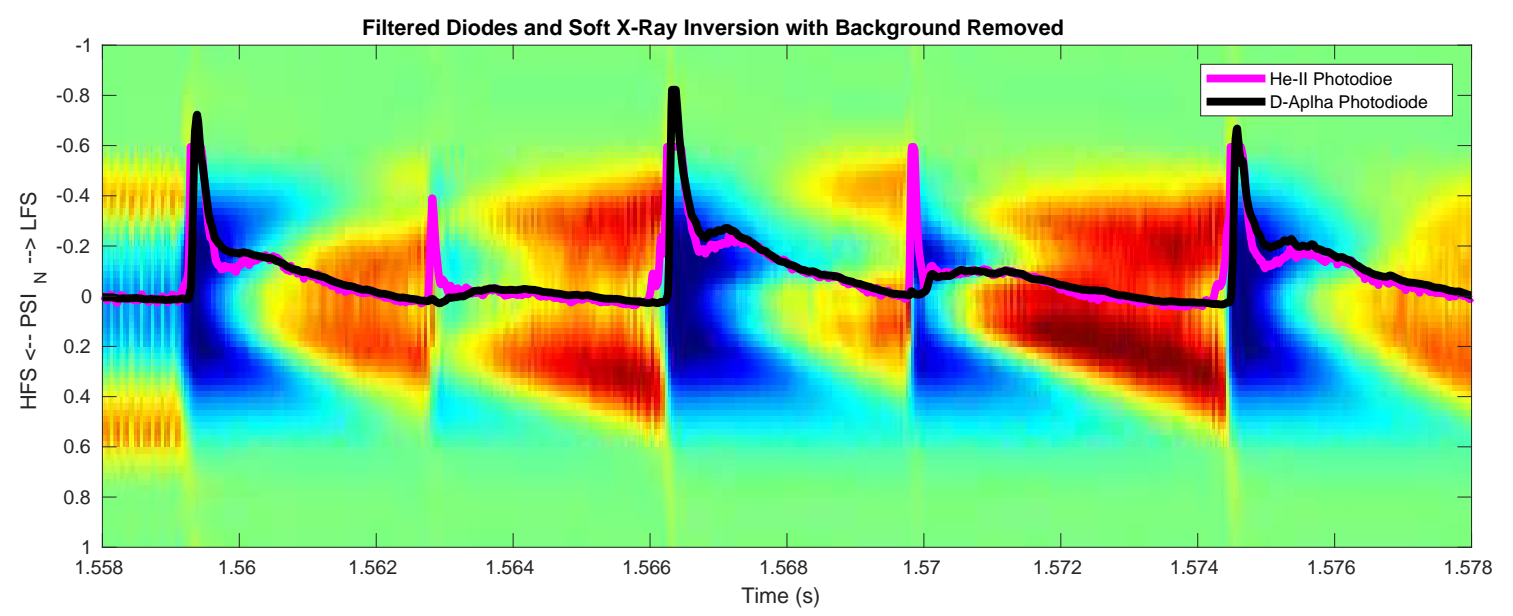

Figure 6. SXR radiation reconstruction from the DMPX diagnostic overlaid with the D-alpha and He-II filtered diode signals for discharge 59183.

The large and small minor disruptions had different effects on the plasma. The global, large minor disruptions caused rapid plasma position movements with the internal, small minor disruptions producing smaller, slower, changes. The LIUQE reconstructed magnetic axis position demonstrates these changes in Figure 7. Simultaneous spikes in the D-alpha and He-II filtered photodiodes are characteristic of the large minor disruptions and spikes only in the He-II photodiode indicate the smaller, core confined minor disruptions. A fast inward movement of the plasma coupled with a vertical oscillation was observed for each large minor disruption whereas only relatively small and slower changes were seen during the internal small minor disruptions.

The disruptive phase of the plasma was maintained for up to $0.7 \mathrm{~s}$ and the plasma then terminated either by a technical disruption such as saturation of the $\mathrm{OH}$ coil or gyrotron shut off, or a terminating disruptive event. The reference discharge presented in this section produced a total of 54 large and 132 small minor disruptions as shown in Figure 8. On average, the large minor disruptions resulted in a loss of over $50 \%$ in 

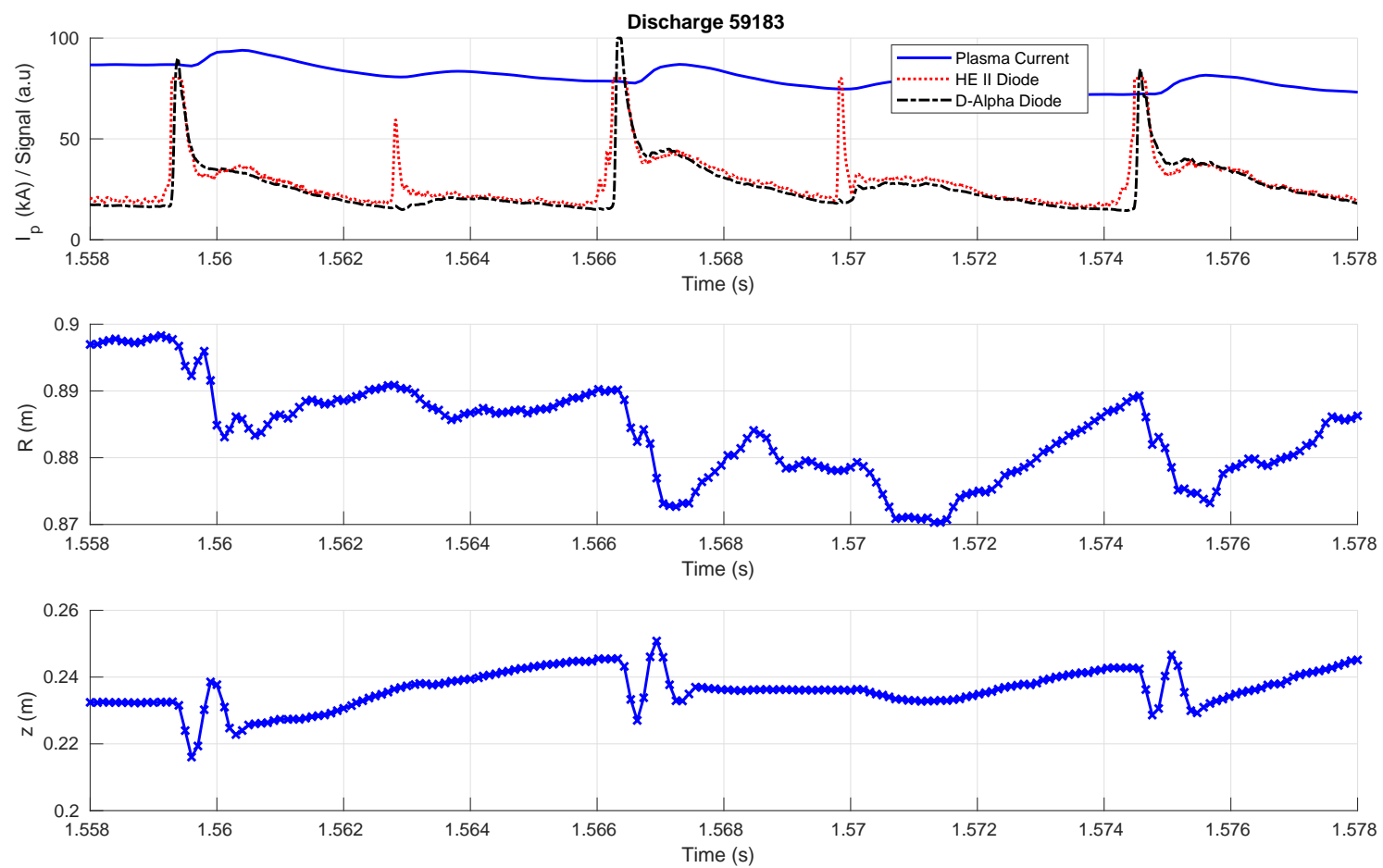

Figure 7. Plasma current, D-alpha and He-II filtered diode signals indicating the minor disruptions and the corresponding change in magnetic axis calculated from LIUQE.

stored energy with the small minor disruptions losing approximately $25 \%$. This scenario was highly reproducible and produced numerous minor disruptions for each discharge allowing for the development of identification and avoidance systems.

\section{Experimental Results}

The experimental results of these methods are presented in four sections; locked mode prevention, suppression of the disruptive phase, ramp down scenarios and testing of the complete closed loop system. The locked mode prevention subsection utilizes radiation intensity triggers signalling the influx of impurities and was used to try to act before the NTM is able to grow and become disruptive. The suppression of the disruptive phase subsection investigates actions taken after the NTM has become disruptive and a locked-mode detector trigger is sent to the control system. Ramp down scenarios were experimentally tested to generate 'soft-landings' or safe terminations of the discharge with rotating and/or disruptive NTMs still present. A complete RT closed loop control procedure was developed from these experiments and incorporated into the TCV control system for testing. A final results section presents the performance of this system, which was tested with increasing quantities of injected gas to simulate more challenging disruption scenarios. 

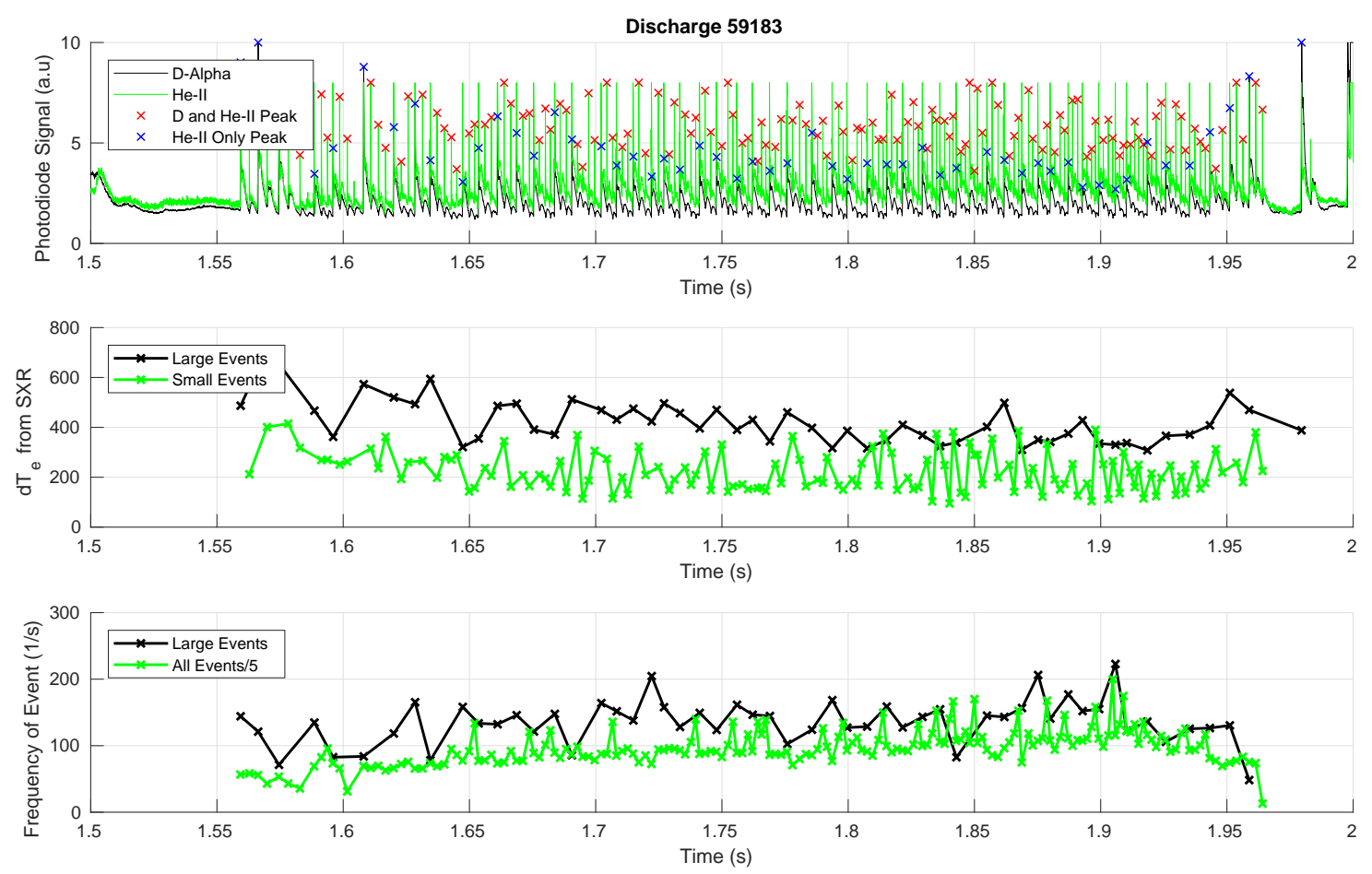

Figure 8. Top - Identification of small and large minor disruptions (events) inffered from filtered photodiode measurements. Middle - Change in core $T_{e}$ from the xTe diagnostic. Bottom - Frequency of events.

\subsection{Locked Mode Prevention}

Impurity influxes produce unique radiation intensity signatures that can be used to provide information to a control system. The SXR intensity measured using the central four DMPX chords was used as a trigger in this series of experiments. The trigger level was based on the disruptive reference at more than double the SXR radiation measured during steady operation. Based on previous studies, ECCD deposition targeting the $q=2$ surface was selected as the most appropriate disruption avoidance actuator. A low gyrotron power of $100 \mathrm{~kW}$ was selected making any effect more sensitive to deposition location and also avoid changing the target plasma significantly through additional heating. A poloidal launcher angle offset of $3.5^{\circ}$ in each direction was used to simulate a realistic technical uncertainty in alignment with the island. This resulted in peak ECCD deposition on, inside and outside the LIUQE reconstructed $q=2$ surface. The ECCD launcher angle prescribed for $\mathrm{q}=2$ deposition was predetermined using the ray tracing code TORAY-GA [34] and the resulting deposition locations from the scan are presented in Figure 9. The location of the LIUQE reconstructed $q=2$ surface is depicted by the black dashed lines.

Windowed Fourier transform spectra of MHD activity and key plasma parameters are presented for each of the experiments in Figure 10. A sharp decrease in stored energy was measured following gas injection at $1.50 \mathrm{~s}$ and the SXR trigger level was 

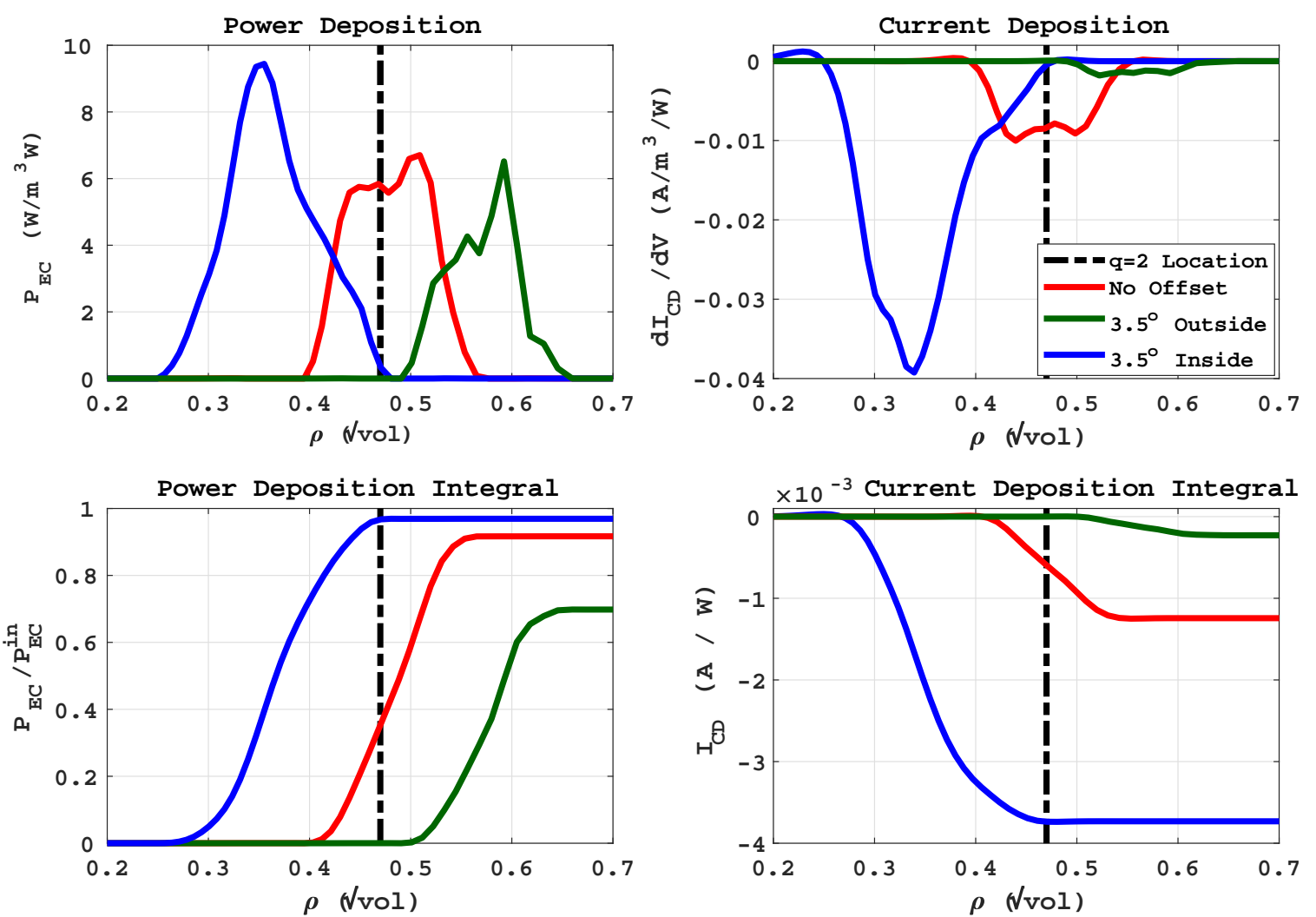

Figure 9. Power and current deposition $10 \mathrm{~ms}$ after gas injection for discharges 59251 , 59250 and 59336 respectively. Black dashed lines indicate the position of the $\mathrm{q}=2$ surface as reconstructed by LIUQE.

attained approximately $10 \mathrm{~ms}$ after gas injection. Peak current deposition outside of the $\mathrm{q}=2$ surface was not effective in preventing mode growth to an unstable amplitude where plasma entered the disruptive phase. Peak current deposition on and slightly inside the computed $q=2$ surface limited the size of the NTM, preventing the onset of the disruptive phase.

Bandpass frequency filtering between $5 \mathrm{kHz}$ and $10 \mathrm{kHz}$ of the SXR radiation measured by the XTOMO diagnostic was employed to remove background radiation from the plasma and the impurity injection. The resulting tomographic inversion of the SXR emission is presented in Figure 11 for a single time slice integrated over $0.1 \mathrm{~ms}$ and overlaid with the $q=2$ surface calculated by LIUQE. The SXR reconstruction suggests that the islands were closer to the core than the LIUQE reconstructed position of the $q=2$ surface. Therefore, peak ECCD deposition inside the LIUQE calculated $q=2$ surface still has finite deposition inside the island, competing with the detrimental effect from the increased bootstrap current and resulting in an overall stabilizing effect. Lack of experimental time did not allow for discharges with deposition clearly inside the island and is left for future studies.

The decrease in mode amplitude and frequency observed with ECCD deposition 

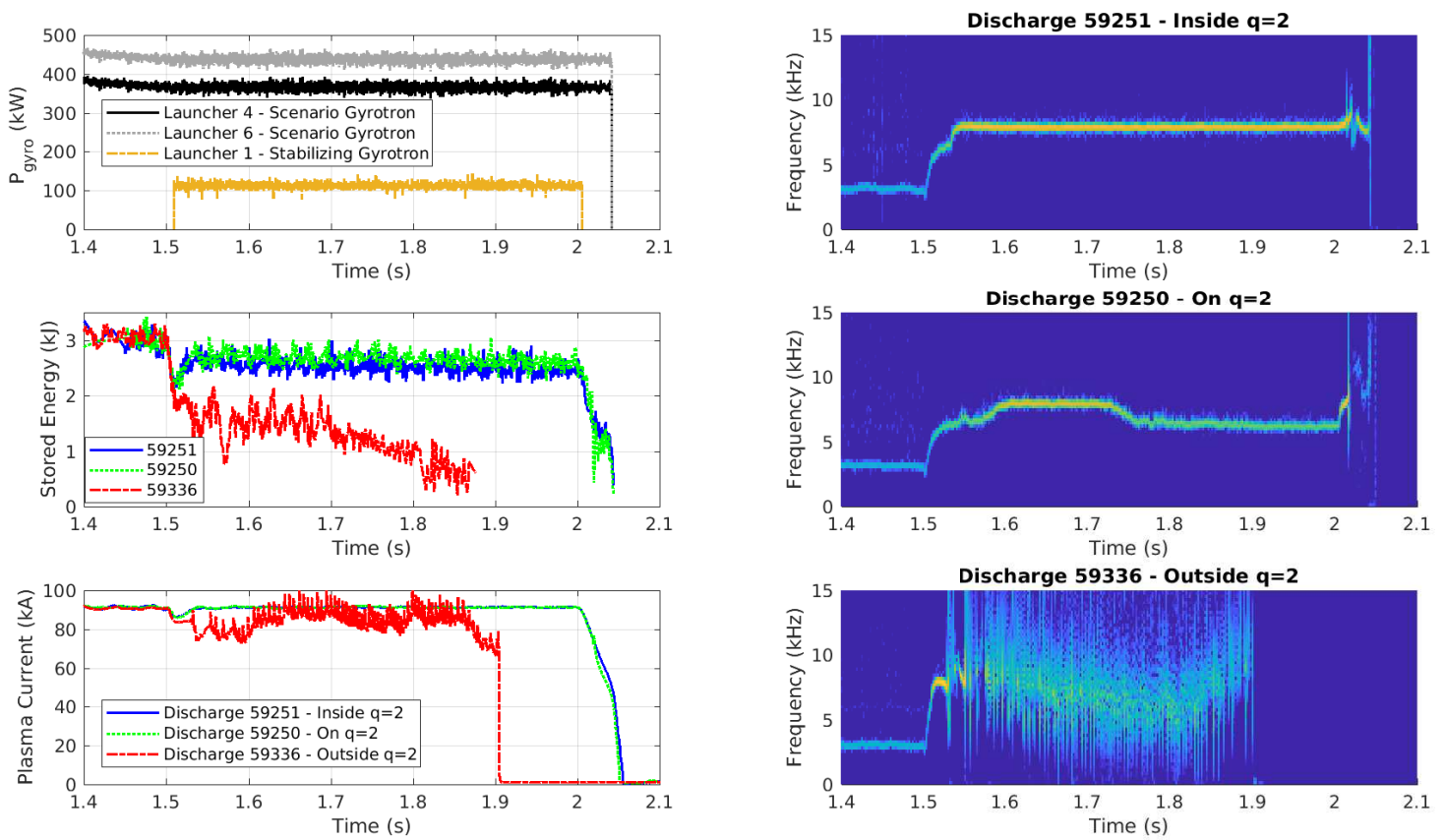

Figure 10. Gyrotron power, stored energy, plasma current and windowed Fourier spectra of MHD activity from locked mode prevention experiments with $100 \mathrm{~kW}$ stabilizing gyrotron power and variation of the deposition radius. The color axis is maintained between the spectra.

on the $\mathrm{q}=2$ surface, relative to inside the $\mathrm{q}=2$ surface, can also be explained by the balance of destabilizing bootstrap current and stabilizing ECCD deposition inside the island. Deposition at the calculated $q=2$ surface may have been offset from the center of the island but there is still finite current deposition within the island and thus a stabilizing effect. Deposition closer to the plasma separatrix resulted in a relatively weaker destabilizing effect from the bootstrap current, leading to an overall lower growth rate than the discharge with peak current deposition inside the calculate $q=2$. In each case, removal of stabilizing power at $2.0 \mathrm{~s}$ caused a fast increase in mode amplitude, leading to a rapid decrease in stored energy and a terminating major disruption. The disruptive effects of removing the stabilizing power confirms the role of continual current drive in the prevention of mode growth.

\subsection{Suppression of the Disruptive Chain}

The sub-ms time scale of the NTM amplitude increase that leads to the disruptive chain did not allow for action to be taken upon the unstable mode that quickly grows. Instead, ECCD was applied continuously during the discharge once the disruptive phase was established in order to exit the disruptive phase, maintain a steady NTM and continue the discharge. Key results are present in Figure 12 for two disruptive phase suppression experiments. The disruptive reference (discharge \# 59183) is also presented 


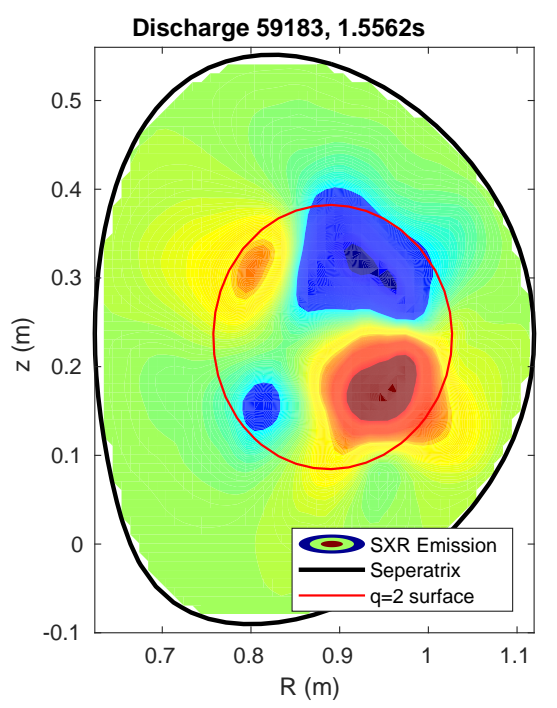

Figure 11. Frequency filtered SXR inversion using the XTOMO diagnostic showing the $\mathrm{m}=2$ mode during the fast rotation and increasing amplitude phase of the discharge.

for comparison. Current drive was applied at approximately $1.7 \mathrm{~s}$ in both disruption avoidance attempts with the plasma exiting the disruptive phase. Approximately $150 \mathrm{~ms}$ of $500 \mathrm{~kW}$ of gyrotron power, providing $1.9 \mathrm{kA}$ of localized current drive, was required to suppress the disruptive chain and establish a stationary rotating NTM until the programmed end of the discharge. A shorter period of $110 \mathrm{~ms}$ was required at a higher gyrotron power of $800 \mathrm{~kW}$, providing $3.0 \mathrm{kA}$ of localized current drive, suppressing the disruptive chain and completely stabilizing the NTM. These results are in good agreement with Kong 2017, which found that a gyrotron power of $750 \mathrm{~kW}$ was required to completely stabilize an NTM in a similar scenario without gas injection [13].

Additional experiments were performed to investigate the sensitivity of the deposition location on the stabilization of the disruptive chain. QI-ECE was used to track the position of the saturated NTM and provide a launcher angle to the ECCD system. The computed angles from this measurement were compared with experiments conducted using RT-TORBEAM and preprogrammed trajectories using TORAY. The RT-TORBEAM code and QI-ECE system were not able to track and deposit current drive on the $q=2$ surface within the disruptive phase due to the fast current profile changes rapidly displacing the $q=2$ surface. To overcome this limitation, the control system was programmed to lock the ECCD launcher angle at the value computed $100 \mathrm{~ms}$ prior to the locked mode trigger signal. This allowed the ECCD current to be deposited at the $\mathrm{q}=2$ surface for a recovering plasma aiming at preventing further disruptive events once the plasma had recovered sufficiently. The current drive deposition location could only be reliably calculated once the plasma had exited the disruptive phase and Table 1 presents these results. The location of the q-surface for this study was estimated using the LIUQE code and the deposition location was calculated using TORAY. It should be noted that the temporal resolution of this code is limited by the measurement of 

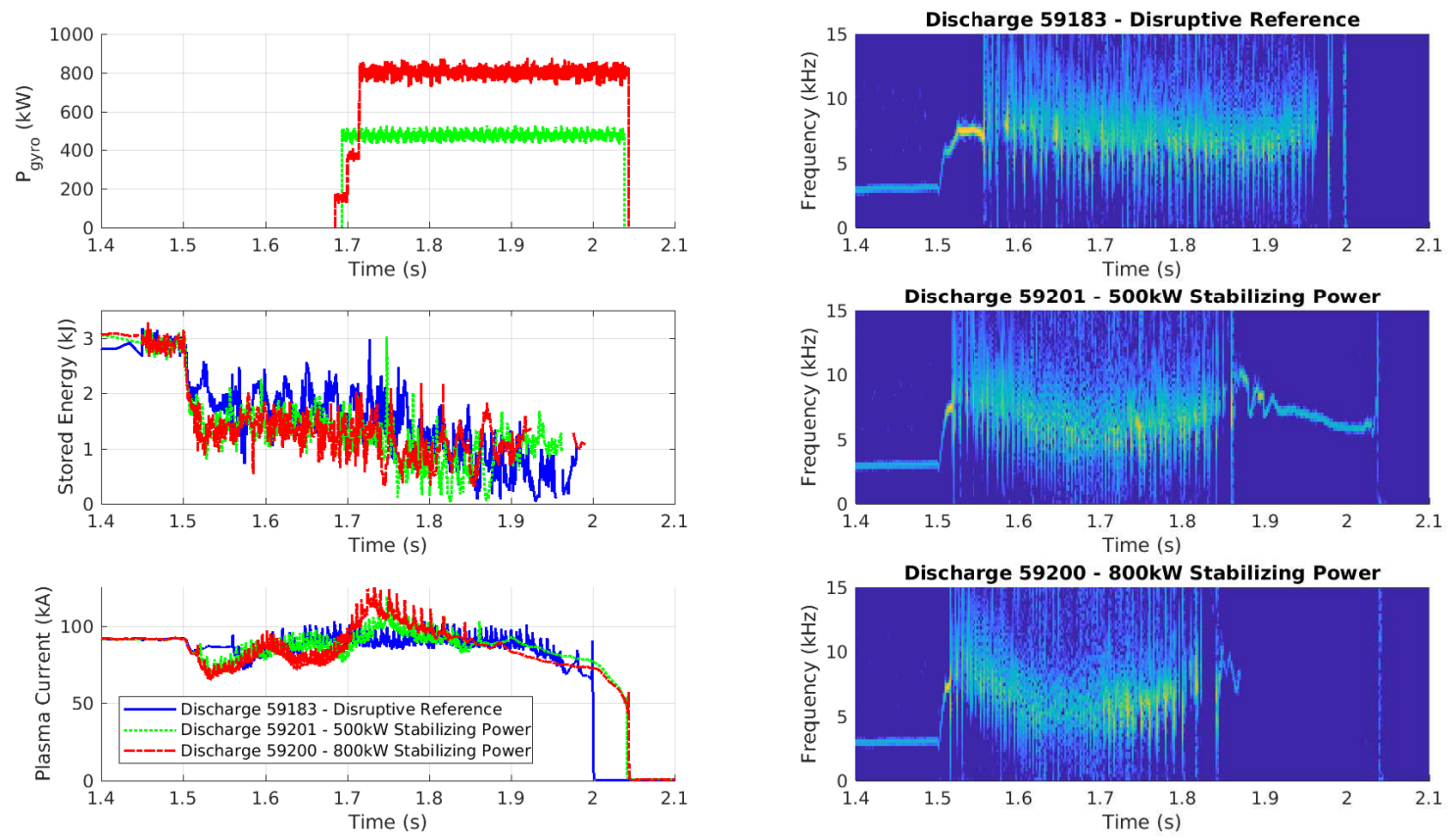

Figure 12. Results of suppression discharges with stabilising gyrotron power, stored energy, plasma current and windowed Fourier spectra of MHD activity from suppression of disruptive chain experiments.

Table 1. Current drive deposition location at time of suppression of disruptive chain and launcher control system.

\begin{tabular}{ccc}
\hline Discharge & Deposition Location (q) & Control System \\
\hline 58680 & 1.9 & QI-ECE \\
59191 & 2.2 & Feed Forward Fixed \\
59193 & 1.9 & Feed Forward Sweep \\
59194 & 1.9 & Feed Forward Sweep \\
59197 & 2.1 & Feed Forward Sweep \\
59199 & 2.1 & TORBEAM \\
59200 & 2.1 & TORBEAM \\
59201 & 2.2 & Feed Forward Fixed \\
59202 & 2.1 & Feed Forward Fixed \\
\hline
\end{tabular}

electron temperature and density profiles, which in this study was $17 \mathrm{~ms}$ as dictated by the Thomson Scattering repetition rate. The deposition location at the suppression of disruptive chain indicates a strong q-surface sensitivity. Discharges were recovered when power was deposited at the $\mathrm{q}=2$ surface $\pm 10 \%$. Both RT systems, RT-TORBEAM and QI-ECE, yielded similar results and were able to deposit ECCD close to the reconstructed location of the $q=2$ surface. 


\subsection{Ramp Down Scenarios}

A complete disruption avoidance system requires a safe termination of the discharge. To this end, experiments were performed to test current ramp rates, gyrotron power sequences and shape variation and their effect on the minimum plasma current that could be attained before a final disruption. Four scenarios were attempted:

- Discharge 59203 - $200 \mathrm{~ms} I_{p}$ ramp down in the disruptive phase with simultaneous gyrotron power ramp down, $55 \mathrm{kA}$ at disruption

- Discharge 59247 - $200 \mathrm{~ms} I_{p}$ ramp down prior to the disruptive phase with simultaneous gyrotron power ramp down, $50 \mathrm{kA}$ at disruption

- Discharge 59187 - $50 \mathrm{~ms} I_{p}$ ramp down after a reduction in elongation in the disruptive phase with constant gyrotron power, $40 \mathrm{kA}$ at disruption

- Discharge 59246 - $50 \mathrm{~ms} I_{p}$ ramp down prior to the disruptive phase with simultaneous gyrotron power ramp down, $40 \mathrm{kA}$ at disruption

In all four scenarios plasma current was reduced to at least $55 \mathrm{kA}$ before the disruption and the results are presented in Figure 13. The highest reduction before a disruption was achieved with the $50 \mathrm{~ms}$ ramp down. It may have been possible to further reduce the final current by simultaneous reduction in elongation but this was not tested as lower plasma currents are currently not possible on TCV due to control system limitations. Furthermore, reducing the time required to modify the shape before a ramp down increased the temporal margin to disruption and therefore a direct $50 \mathrm{~ms}$ current ramp down was the preferred option from those tested.
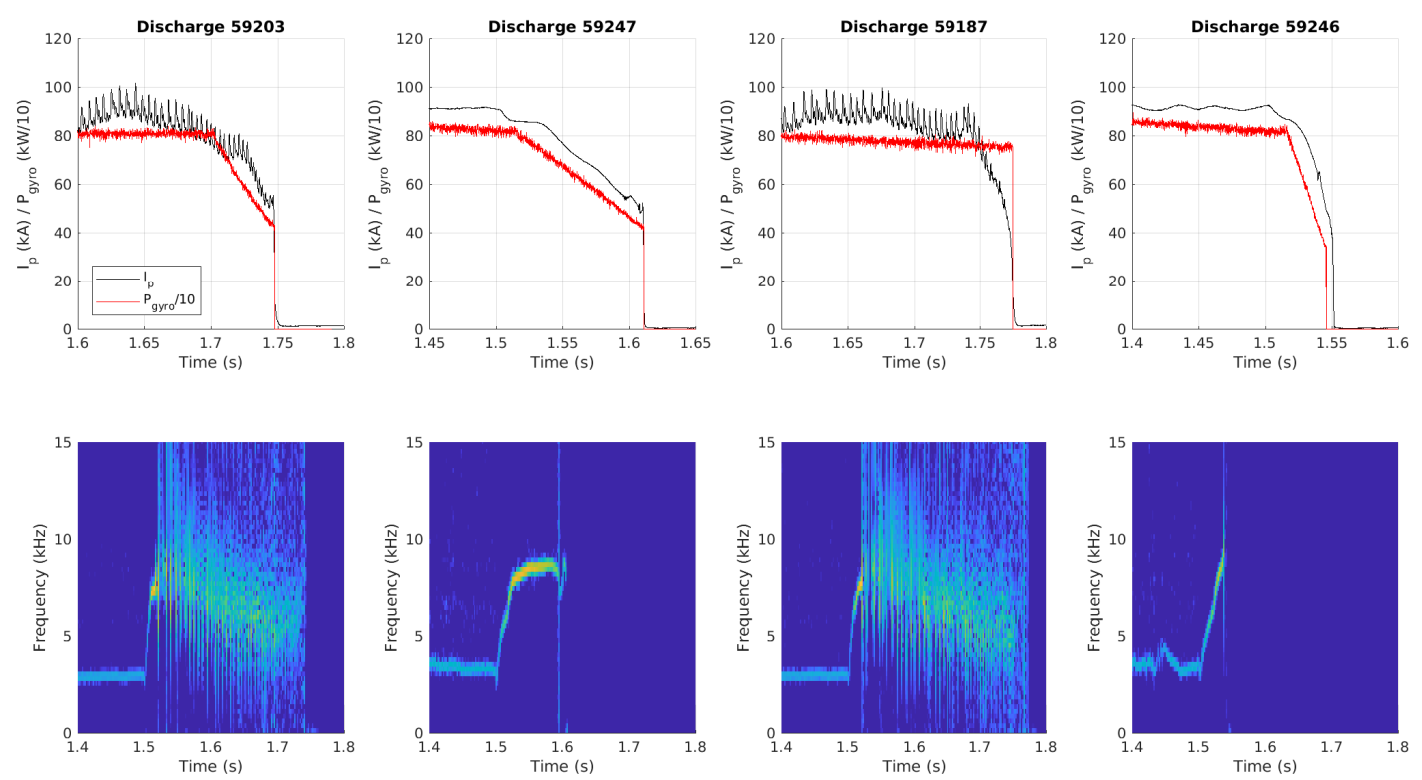

Figure 13. Total gyrotron power, plasma current and windowed Fourier spectra of MHD activity for ramp down discharges described in Section 3.3. 


\subsection{Closed Loop Testing}

The experiments presented above were used to design a closed loop system to handle the disruptive scenario. The RT control system utilized RT-LIUQE and RT-TORBEAM to continually track and target the $\mathrm{q}=2$ surface throughout the discharge. RT-TORBEAM was preferred to QI-ECE as it operates in the absence of a rotating mode, a prerequisite for the QI-ECE system. The locked mode and SXR triggers were simultaneously monitored by the control system. Maximum available gyrotron power was provided to the launcher targeting the $q=2$ surface when either the SXR or locked mode detector threshold was reached. The ECCD launcher angle was held constant after a locked-mode trigger until the locked-mode trigger signal returned below another threshold for more than $50 \mathrm{~ms}$, after which the control system restarted to track and target the $\mathrm{q}=2$ surface. In the case of a SXR trigger and no locked mode, the control system tracking and $q=2$ surface targeting was maintained to prevent the mode from destabilizing. This situation was programmed to be maintained for $200 \mathrm{~ms}$ to demonstrate continued prevention of the NTM destabilizing before a $50 \mathrm{~ms}$ ramp down of plasma current and gyrotron power.

After successful testing, the injected gas quantity was increased to test the efficacy of the disruption avoidance system. Figure 14 presents experiments with double and quadruple the injected gas quantities, 1.6 mbar L and $3.2 \mathrm{mbar} \mathrm{L}$ respectively. A 'no action' reference is also presented for comparison. In both tests the mode induced fast decrease in stored energy was halted and the mode did not lock. The plasma stored energy was restored to pre-impurity influx levels due to a combination of mode stabilization and the balance of the incoming off-axis gyrotron power and the increase in radiated power. A spinning mode was maintained for $200 \mathrm{~ms}$ before the plasma current ramp down which then disrupted at $40 \mathrm{kA}$ in both discharges. This was the optimum achievable result with the present limitations of TCV's control system.

\section{Conclusions}

This study was able to develop a path-orientated disruption handling system by first creating a relevant scenario for high performance discharges, developing avoidance techniques and applying a closed loop control scenario that was able to extend an unhealthy discharge and then safely terminate it. The dataset created through this work provides a direct comparison of possible triggers that can be employed to diagnose, and in the future, predict this specific type of disruption. The closed loop system demonstration was conducted with increasingly challenging disruptive scenarios and was able to recover the discharge and produce a safe termination in each case. It must be stressed that the range of disruptions treated in these experiments is presently limited but this approach could be envisaged for other disruption provoking situations. Nevertheless it concerns discharges with NTMs and impurity accumulation which is relevant for many present scenarios.

Theoretical and empirical transport scaling modelling of the interactions between 

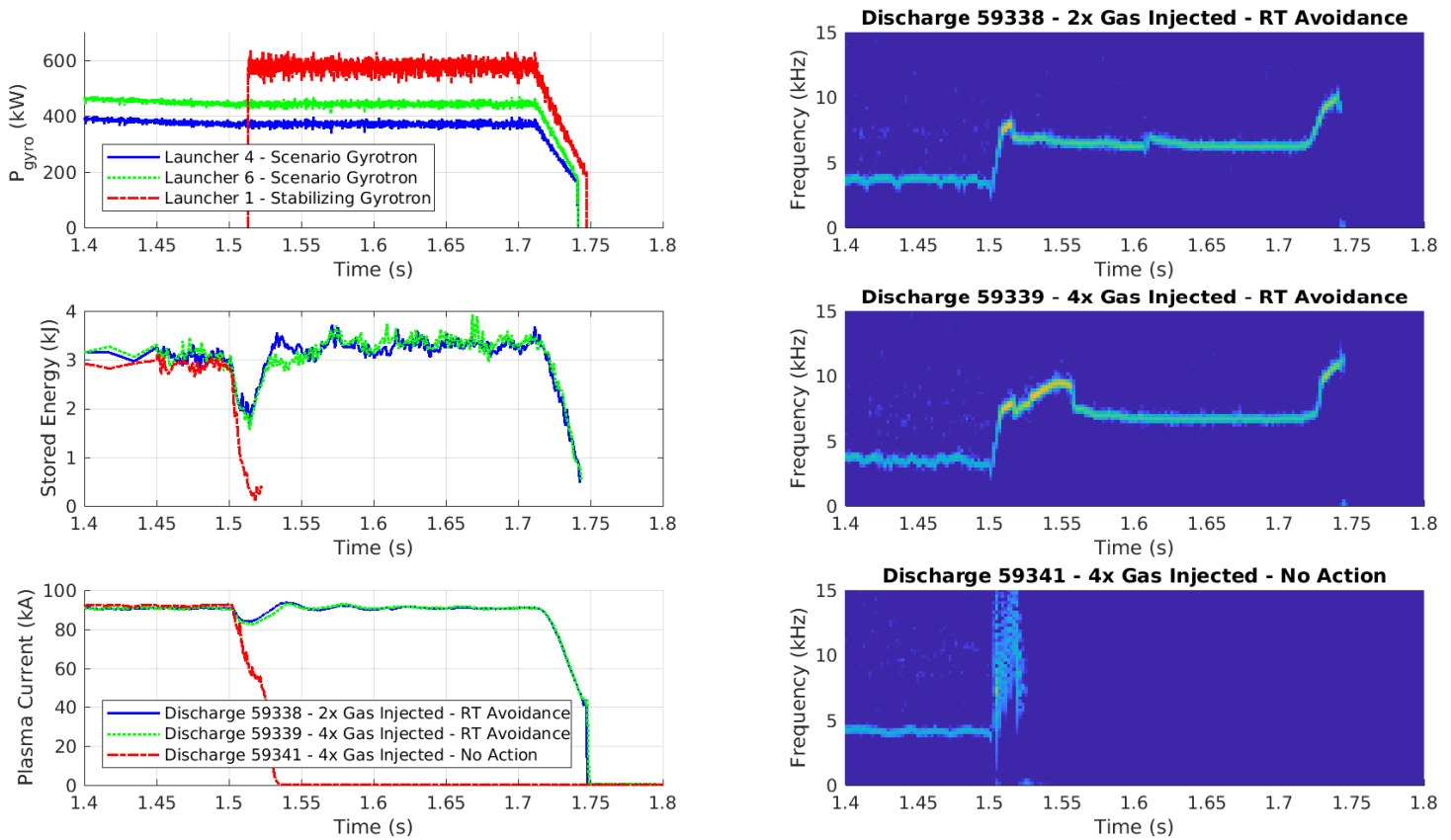

Figure 14. Gyrotron power, stored energy, plasma current and windowed Fourier spectra of MHD activity for discharges with the avoidance control system and a reference with no action.

ECCD/ECRH and NTMs observed in these experiments is planned so that the physics can be implemented and extrapolated to other experiments, and in particular to future experiments such as ITER. This work will enable more global constraints on the deposition location accuracy and amount of current drive required to prevent NTM destabilization or react so as to control such an NTM and terminate the discharge safely. A more detailed study of any difference between current drive and ECRH heating is planned for the near future to better identify the stabilisation physics involved. Finally, the relatively long lived disruption vulnerable discharge scenario developed in this work serves as a reliable target for new approaches to plasma state monitoring and disruptive plasma management.

\section{Acknowledgements}

This work has been carried out within the framework of the EUROfusion Consortium and has received funding from the Euratom research and training programme 20142018 under grant agreement No 633053. The views and opinions expressed herein do not necessarily reflect those of the European Commission. This work was supported in part by the Swiss National Science Foundation. The authors would like to thank the MST high level topic 9 team for collaboration on the QIE system. 


\section{Bibliography}

[1] De Vries, P.C., et al. 2011 Nucl. Fusion 51053018

[2] De Vries, P.C., et al. 2014 Phys. Plasmas 21056101

[3] Pautasso, G., et al. 2014 Proceedings of the 41st European Physics Conference on Plasma Physics, vol 38F, Berlin Germany.

[4] Maraschek, M., et al. 2018 Plasma Phys. Control. Fusion 60014047

[5] Sauter, O., et al 2010 Plasma Phys. Control. Fusion 52025002

[6] Maraschek, M., 2012 Nucl. Fusion 52074007

[7] Gantenbein, G., et al 2000 Phys. Rev. Lett. 85(6), 1242(4)

[8] Urso, L., et al 2010 Nucl. Fusion 50025010

[9] Felici, F., et al. 2012 Nucl. Fusion 52074001.

[10] Choi, W., et al. 2018 Nucl. Fusion 58036022

[11] Gates, D.A., et al. 1997 Nucl. Fusion 371593

[12] Westerhof, E., et al. 2002 Nucl fusion 421324

[13] Kong, M., et al. 2017 44th EPS Conference on Plasma Physics.

[14] Zohm, H., 1997 Phys. Plasmas 43433

[15] Hegna, C. C., et al. 1997 Phys. Plasmas 42940

[16] Peltzer, A., et al. 1999 Phys. Plasmas 61589

[17] Zohm, H., et al. 2001 Nucl. Fusion 41197

[18] Hoshino, K., et al 1992 Phys. Rev. Lett. 692208

[19] Kislov, D. A., et al 1997 Nucl. Fusion 37339

[20] Savrukhin, P. V., et al 1994 Nucl. Fusion 34317

[21] Salzedas, F., et al 2002 Nucl. Fusion 42881

[22] Esposito, B., et al 2008 Phys. Rev. Lett. 100045006

[23] Maraschek, M., 2005 Nucl. Fusion 451369

[24] La Haye, R. J., 2006 Phys. Plasmas 13(5):055501

[25] Maraschek, M., et al. 2007 Physical review letters 98025005.

[26] Isayama, A., et al 2009 Nucl. Fusion 49055006

[27] Poli, E., Peeters, A. G., and Pereverzev, G. V., 2001 Computer Physics Communications 136.12:90-104

[28] Galperti, C., et al. submitted 2018 30th Symposium on Fusion Technology

[29] Rispoli, N., et al. submitted 2018 30th Symposium on Fusion Technology

[30] Moret, J-M., et al. 2015 Fusion Engineering and Design 91:1-15.

[31] Coda, S., et al. 2017 Nucl. Fusion 57102011

[32] Moret, J-M., et al. 1998 Review of Scientific Instruments 69, 23332348. doi: 10.1063/1.1148940

[33] Piras, F., 2011 Extremely shaped plasmas to improve the Tokamak concept, retrieved from EPFL Infoscience (10.5075/epfl-thesis-5015)

[34] Matsuda, K., et al. 1989 IEEE Trans. Plasma Scien., 17(1):6 